\title{
NOVA SOLUÇÃO ELETROLÍTICA INTRAVENOSA PARA USO VETERINÁRIO: EFEITOS SOBRE O PERFIL ELETROLÍTICO E ÁCIDO BASE EM BOVINOS ADULTOS HÍGIDOS
}

\section{EFFECTS OF A NEW INTRAVENOUS ELECTROLYTE SOLUTION FOR USE IN VETERINARY PRACTICE ON THE ELECTROLYTE AND ACID-BASE BALANCES OF HEALTHY CATTLE}

\author{
Priscilla Fajardo Valente Pereira ${ }^{1^{*}}$ \\ José Antonio Bessegatto ${ }^{1}$ \\ Gabriela de Castro Bregadioli ${ }^{1}$ \\ José Guilherme do Rego Marcondes ${ }^{1}$ \\ Thiago Marquez Fernandes ${ }^{1}$ \\ Mara Regina Stipp Balarin ${ }^{1}$ \\ Karina Keller Marques da Costa Flaiban ${ }^{1}$ \\ Júlio Augusto Naylor Lisbôa ${ }^{1}$ \\ 1Universidade Estadual de Londrina, Londrina, PR, Brasil \\ *Autora para contato - pfajardo@uel.br
}

\section{Resumo}

Objetivou-se avaliar os efeitos de uma nova solução hidratante intravenosa sobre os equilíbrios eletrolítico e ácido-base de bovinos. Para tanto, oito animais adultos hígidos, mestiços taurinos foram submetidos a dois tratamentos em delineamento cross-over: solução salina isotônica (SI) e solução teste (ST) composta por $145 \mathrm{mEq}$ de $\mathrm{Na}^{+}, 5 \mathrm{mEq}$ de $\mathrm{K}^{+}, 4 \mathrm{mEq} \mathrm{de} \mathrm{Ca}^{++}, 2 \mathrm{mEq}$ de $\mathrm{Mg}^{++}, 96 \mathrm{mEq}$ de $\mathrm{Cl}^{-}, 60 \mathrm{mEq}$ de lactato, $50 \mathrm{~g}$ de dextrose e $4 \mathrm{mg}$ de cianocobalamina por litro. As soluções foram administradas por via intravenosa, em volume correspondente a $5 \%$ do peso corporal, durante três horas de infusão contínua. Amostras de sangue venoso foram colhidas antes da infusão e três, seis, nove e 24 horas após o início da infusão para determinações de glicose, lactato $\mathrm{L}, \mathrm{Ca}, \mathrm{P}, \mathrm{Mg}, \mathrm{pH}$, $\mathrm{pCO}_{2}, \mathrm{HCO}_{3}{ }^{-}, \mathrm{BE}, \mathrm{Na}^{+}, \mathrm{K}^{+}, \mathrm{Cl}^{-}$e $\mathrm{Ca}^{++}$. Os resultados foram analisados por análise de variâncias de medidas repetidas. Ao contrário da SI, que causou hipercloremia e redução da diferença de íons fortes no plasma (SID), determinando efeito acidificante, a ST gerou alcalinização leve e transitória e não provocou alteração das concentrações de $\mathrm{Na}^{+}$e de $\mathrm{Cl}^{-}$. A hipopotassemia e a hiperglicemia, presentes ao final da infusão da ST, foram transitórias e não determinaram sinais clínicos evidentes. Concluise que a ST é uma alternativa segura para a terapia com fluidos em bovinos, tendo a vantagem de provocar alterações iatrogênicas de baixa magnitude.

Palavras-chave: gasometria, eletrólitos, terapia com fluidos intravenosa.

\section{Abstract}

The effects of a new intravenous polyionic solution on electrolyte and acid-base balances in cattle 
were evaluated. Eight healthy adult, crossbred Bos taurus cattle received two treatments in a crossover design: isotonic saline solution (IS) and test solution (TS) with $145 \mathrm{mEq}$ of $\mathrm{Na}^{+}, 5 \mathrm{mEq}$ of $\mathrm{K}^{+}$, $4 \mathrm{mEq}$ of $\mathrm{Ca}^{++}, 2 \mathrm{mEq}$ of $\mathrm{Mg}^{++}, 96 \mathrm{mEq}$ of $\mathrm{Cl}^{-}, 60 \mathrm{mEq}$ of lactate, $50 \mathrm{~g}$ of dextrose, and $4 \mathrm{mg}$ of cyanocobalamin per liter. The solutions were IV infused in a volume corresponding to $5 \%$ of $\mathrm{BW}$, through 3 hours. Venous blood samples were taken before the infusion and 3, 6, 9, and 24 hours after the beginning of the infusion. Glucose, L-lactate, $\mathrm{Ca}, \mathrm{P}, \mathrm{Mg}, \mathrm{pH}, \mathrm{pCO}_{2}, \mathrm{HCO}_{3}{ }^{-}, \mathrm{BE}, \mathrm{Na}^{+}, \mathrm{K}^{+}, \mathrm{Cl}^{-}$, and $\mathrm{Ca}^{++}$were measured. Data were analyzed through repeated measures ANOVA. IS caused acidifying effect due to hyperchloremia and decreased plasma strong ion difference (SID). On the contrary, TS induced mild and transient alkalinization without changes in $\mathrm{Na}^{+}$and $\mathrm{Cl}^{-}$plasma levels. Transient hypokalemia and hyperglycemia were presented at the end of the ST infusion but did not provoke apparent clinical signs. We concluded that TS is a safe choice for fluid therapy in cattle, inducing low magnitude iatrogenic changes as an advantage.

Keywords: blood gas analyses, electrolytes, intravenous fluid therapy.

Recebido em: 13 novembro de 2014

Aceito em: 13 dezembro 2016

\section{Introdução}

A terapia com fluidos é imprescindível para melhorar a perfusão sanguínea e a oxigenação tecidual, corrigir os desequilíbrios hídrico, eletrolítico e ácido-base, fornecer suporte energético e reverter o choque hipovolêmico $^{(1)}$. Em muitas situações, a administração oral ou enteral de fluidos contendo eletrólitos é preferida, porque é econômica e de execução fácil ${ }^{(2)}$. No entanto, o trânsito aboral preservado da ingesta no aparelho digestório é a condição para que essa via de administração possa ser adotada. Quando o movimento aboral está retardado ou impedido, a via intravenosa torna-se obrigatória $^{(3)}$. Além disso, a administração intravenosa de fluidos é indispensável em determinadas enfermidades que acometem os ruminantes, como septicemia e endotoxemia, distúrbios metabólicos graves e desidratações acentuadas ${ }^{(4)}$.

As soluções cristaloides poliônicas que reúnem características ideais para a infusão intravenosa veiculam variedade relativamente pequena de eletrólitos e são seguras para a administração de grandes volumes, especialmente, se possuírem osmolaridade parecida com a do plasma ${ }^{(1)}$. As soluções tradicionalmente empregadas em medicina, tais como a salina isotônica ( $\mathrm{NaCl} 0,9 \%)$, a de Ringer simples, a de Ringer com lactato (SRL) e a glicofisiológica, possuem essas características sendo utilizadas com sucesso em ruminantes ${ }^{(1)}$.

Tais soluções podem interferir nas concentrações dos eletrólitos no líquido extracelular provocando efeito acidificante ou alcalinizante iatrogênicos, dependendo de sua composição, da velocidade de infusão e do volume administrado ${ }^{(1,5)}$. Por esse motivo, enquanto as soluções de bicarbonato de sódio $\left(\mathrm{NaHCO}_{3}\right)$ são apropriadas para a correção da acidose metabólica ${ }^{(6)}$, outras opções são mais adequadas para a administração em ruminantes com alcalose metabólica ( $\mathrm{NaCl}$ 0,9\%, Ringer simples e Glicofisiológica $)^{(1)}$. Por ser balanceada, a SRL não interfere nos equilíbrios eletrolítico e ácidobase $^{(7)}$, podendo ser administrada com segurança em ambos os desequilíbrios metabólicos.

Dentre os produtos farmacêuticos veterinários disponíveis no Brasil, há poucas opções de soluções 
eletrolíticas intravenosas, ainda que todas sejam indicadas para uso em ruminantes. De forma geral, as soluções disponíveis são hiperosmolares, ou seja, possuem combinação complexa de solutos e concentração elevada de glicose. Essas características, somadas ao preço elevado, tornam proibitiva a infusão de volumes maiores, necessários para a hidratação de animais de maior porte como os bovinos adultos. $\mathrm{O}$ desenvolvimento de soluções eletrolíticas adequadas para a prática mais correta e moderna da terapia com fluidos em bovinos é altamente desejável.

Quando uma nova solução é desenvolvida, os estudos preliminares que verificam a segurança e os desequilíbrios iatrogênicos que poderiam ocorrer são obrigatoriamente realizados em animais saudáveis ${ }^{(8)}$. As investigações sobre eficácia terapêutica devem ser, pelo contrário, realizadas em animais que apresentam desequilíbrios. O objetivo deste estudo foi avaliar os efeitos de uma nova solução eletrolítica administrada por via intravenosa sobre o perfil eletrolítico e ácido-base de bovinos adultos hígidos.

\section{Material e Métodos}

O protocolo experimental foi julgado e aprovado pela Comissão de Ética no Uso de Animais da Universidade Estadual de Londrina (CEUA/UEL), registrado como processo $n^{\circ}$ 2361.2013.16. Todos os procedimentos experimentais foram realizados no Hospital Veterinário da UEL.

A solução testada possuía a seguinte composição: $145 \mathrm{mEq} / \mathrm{L}$ de sódio $\left(\mathrm{Na}^{+}\right), 5 \mathrm{mEq} / \mathrm{L}$ de potássio $\left(\mathrm{K}^{+}\right), 4 \mathrm{mEq} / \mathrm{L}$ de cálcio $\left(\mathrm{Ca}^{++}\right), 2 \mathrm{mEq} / \mathrm{L}$ de magnésio $\left(\mathrm{Mg}^{++}\right), 96 \mathrm{mEq} / \mathrm{L}$ de cloreto $\left(\mathrm{Cl}^{-}\right)$e $60 \mathrm{mEq} / \mathrm{L}$ de lactato, além de $50 \mathrm{~g}$ de dextrose por litro. Uma ampola contendo $2 \mathrm{mg}$ de cianocobalamina foi adicionada a cada frasco de $500 \mathrm{~mL}$ da solução no momento da infusão. A osmolaridade calculada desta solução foi de $562 \mathrm{mOsm} / \mathrm{L}$, semelhante à osmolaridade da solução glicofisiológica. Essa solução possui a mesma composição daquela utilizada por Pereira et al. ${ }^{(9)}$ em equinos e ainda não está disponível no comércio, recebendo a denominação preliminar de Repositor Hidroeletrolítico Vetoquinol (Vétoquinol/Fagra ${ }^{\circledR}$, São Paulo, SP).

Participaram do experimento oito bovinos adultos, mestiços taurinos, quatro machos e quatro fêmeas, saudáveis, pesando de 230 a $640 \mathrm{~kg}$. As fêmeas não estavam gestantes nem em lactação. Os animais eram mantidos em piquete de grama estrela africana (Cynodon nlemfuensis) e recebiam silagem de sorgo em cocho (6 a 8kg/animal/dia), com livre acesso à água e suplementação mineral ad libitum.

Cada bovino foi submetido a dois tratamentos em delineamento experimental cross-over, sendo a ordem dos tratamentos estabelecida por meio de sorteio, com intervalo de cinco dias entre os tratamentos. No tratamento controle (SI), os animais receberam a solução salina isotônica $(\mathrm{NaCl}$ 0,9\%) comercial (Fisiológico, Linhamax ${ }^{\circledR}$, Eurofarma, São Paulo, SP) e no tratamento teste (ST), os animais receberam a solução descrita anteriormente. Foi empregado cateter $18 \mathrm{G}$ (Angiocath ${ }^{\circledR}, \mathrm{BD}$, São Paulo, SP) para fixação na veia auricular e as soluções foram infundidas com velocidade entre 15 e $20 \mathrm{~mL} / \mathrm{kg} / \mathrm{h}$, em três horas de administração contínua, totalizando o volume correspondente a $5 \%$ do peso corporal. Para que a velocidade requerida fosse alcançada, houve necessidade de se realizar a infusão simultânea em duas veias auriculares (uma em cada orelha).

Exames físicos foram realizados nos animais a cada hora durante a infusão intravenosa e nos momentos definidos para a colheita das amostras de sangue, empregando-se os métodos semiológicos 
clássicos $^{(10)}$. O acompanhamento dos animais para observação de comportamento, apetite, atitudes e característica das fezes e da urina foi contínuo por até 24 horas após o início de cada tratamento.

Os métodos empregados são semelhantes àqueles utilizados no estudo de Pereira e colaboradores ${ }^{(9)}$. Amostras de sangue venoso foram colhidas em cinco momentos definidos: imediatamente antes de iniciar a infusão (0h), ao término da infusão (3h), às $6 \mathrm{~h}$, às $9 \mathrm{~h}$ e às $24 \mathrm{~h}$ após o início da infusão. As amostras de sangue foram colhidas por punção da veia jugular com agulhas $21 \mathrm{G}(25 \mathrm{x} 0,8 \mathrm{~mm})$ do sistema de coleta a vácuo, utilizando-se frascos contendo fluoreto de sódio, para as determinações da glicose e do lactato L, e frascos sem anticoagulante, para as determinações de cálcio total (Ca), fósforo (P) e magnésio (Mg). O plasma fluoretado foi obtido por centrifugação das amostras em, no máximo, dez minutos após a colheita e o processamento analítico executado em até oito horas. $\mathrm{O}$ soro sanguíneo foi obtido por centrifugação após a retração do coágulo e conservado por congelação (20 ${ }^{\circ} \mathrm{C}$ negativos) até o momento das análises. Para a hemogasometria, amostras de sangue foram colhidas da veia jugular empregando-se seringas plásticas de $3 \mathrm{~mL}$, contendo cerca de $0,08 \mathrm{~mL}$ (400UI) de heparina sódica como anticoagulante, acopladas a agulhas hipodérmicas $21 \mathrm{G}$ (30 x 0,8mm). As análises foram realizadas em até cinco minutos após a colheita.

Os exames laboratoriais consistiram na determinação das concentrações plasmáticas de lactato L e de glicose e das concentrações séricas de $\mathrm{Ca}, \mathrm{P}$ e $\mathrm{Mg}$, empregando-se métodos colorimétricos (Dimension Clinical Chemistry System; Siemens, São Paulo, SP). O exame hemogasométrico consistiu nas determinações sanguíneas de $\mathrm{pH}$, pressão parcial de dióxido de carbono $\left(\mathrm{pCO}_{2}\right)$, concentração do íon bicarbonato $\left(\mathrm{HCO}_{3}{ }^{-}\right)$, excesso de base $(\mathrm{BE})$ e concentrações de sódio $\left(\mathrm{Na}^{+}\right)$, potássio $\left(\mathrm{K}^{+}\right)$, cloretos $\left(\mathrm{Cl}^{-}\right)$e cálcio ionizado $\left(\mathrm{Ca}^{++}\right)(\mathrm{Omni} \mathrm{C}$, Cobas B121; Roche, São Paulo, SP).

As seguintes variáveis foram calculadas empregando-se as respectivas fórmulas:

a) Hiato aniônico - Anion Gap (AG): $\mathrm{AG}=\left(\mathrm{Na}^{+}+\mathrm{K}^{+}\right)-\left(\mathrm{Cl}^{-}+\mathrm{HCO}_{3}^{-}\right)$.

b) Diferença de íons fortes - Strong Ion Difference (SID): $\mathrm{SID}=\left(\mathrm{Na}^{+}+\mathrm{K}^{+}\right)-\left(\mathrm{Cl}^{-}\right)$.

A análise dos dados foi realizada em etapas distintas. Inicialmente empregou-se análise de variância de medidas repetidas para se avaliar o efeito do fator tempo (antes e após a administração da solução), do fator sexo (machos x fêmeas) e da interação entre os dois fatores. Posteriormente, a mesma análise foi empregada para se testar o efeito do fator tempo (antes e após a administração da solução), do fator tratamento (SI x ST) e da interação entre os dois fatores. Quando a estatística F resultou significativa, o teste de Tukey foi empregado para comparação entre as médias. Admitiu-se a probabilidade de erro de 5\%. O programa SigmaStat for Windows 3.1 foi usado para a realização da análise.

\section{Resultados e Discussão}

O sexo não exerceu efeito sobre as variáveis estudadas $(\mathrm{p}>0,05)$ e não houve interação entre o sexo e o fator tempo $(p>0,05)$. A interação entre o tempo e o tratamento foi significativa para todas as variáveis, exceto $\mathrm{AG}, \mathrm{Ca}, \mathrm{Ca}^{++}$e $\mathrm{Mg}$. O tempo, antes e após a administração das soluções, como fator isolado, provocou efeito em todas as variáveis exceto em $\mathrm{AG}, \mathrm{Ca}, \mathrm{Ca}^{++}$e $\mathrm{Mg}$. $\mathrm{O}$ tipo de solução administrada provocou diferenças nas variáveis estudadas, com exceção de $\mathrm{AG}, \mathrm{Na}^{+}, \mathrm{Ca}, \mathrm{Ca}^{++}, \mathrm{Mg}$ e 
P (Tabela 1).

Com relação ao equilíbrio ácido-base, efeitos antagônicos foram observados após a infusão das duas soluções. A ST gerou alcalinização elevando o $\mathrm{BE}(\mathrm{p}<0,01),{\mathrm{o} \mathrm{HCO}_{3}}^{-}(\mathrm{p}<0,05)$ e o pH $(\mathrm{p}<0,001)$, porém sem provocar alcalemia e sem gerar hipercapnia compensatória (Tabela 1 e Figura 1). Por outro lado, a SI provocou efeito acidificante, caracterizado pela redução do $\mathrm{BE}$ ( $\mathrm{p}<0,01$ ), do $\mathrm{HCO}_{3}{ }^{-}$ $(\mathrm{p}<0,05)$ e do $\mathrm{pH}(\mathrm{p}<0,001)$, porém sem induzir acidemia e sem gerar hipocapnia compensatória, apesar da diminuição da $\mathrm{pCO}_{2}(\mathrm{p}<0,05)$. As soluções provocaram um leve impacto sobre o equilíbrio ácido-base e seus efeitos foram rapidamente corrigidos.

O efeito alcalinizante da ST deve-se à concentração elevada de lactato $(60 \mathrm{mEq} / \mathrm{L})$ na sua composição, mais que o dobro da concentração presente na SRL, que possui $28 \mathrm{mEq} / \mathrm{L}$ deste composto. O lactato administrado é metabolizado pelo organismo, sobretudo no fígado, o que resulta na geração direta e indireta de $\mathrm{HCO}_{3}{ }^{-(1)}$. A administração de soluções que possuem concentrações mais elevadas de lactato $(150 \mathrm{mEq} / \mathrm{L})$ produz alcalinização acentuada ${ }^{(6)}$. A SRL, por outro lado, não causa impacto sobre o equilíbrio ácido-base, como comprovado em equinos, em bezerros e em ovelhas $^{(7)}$.

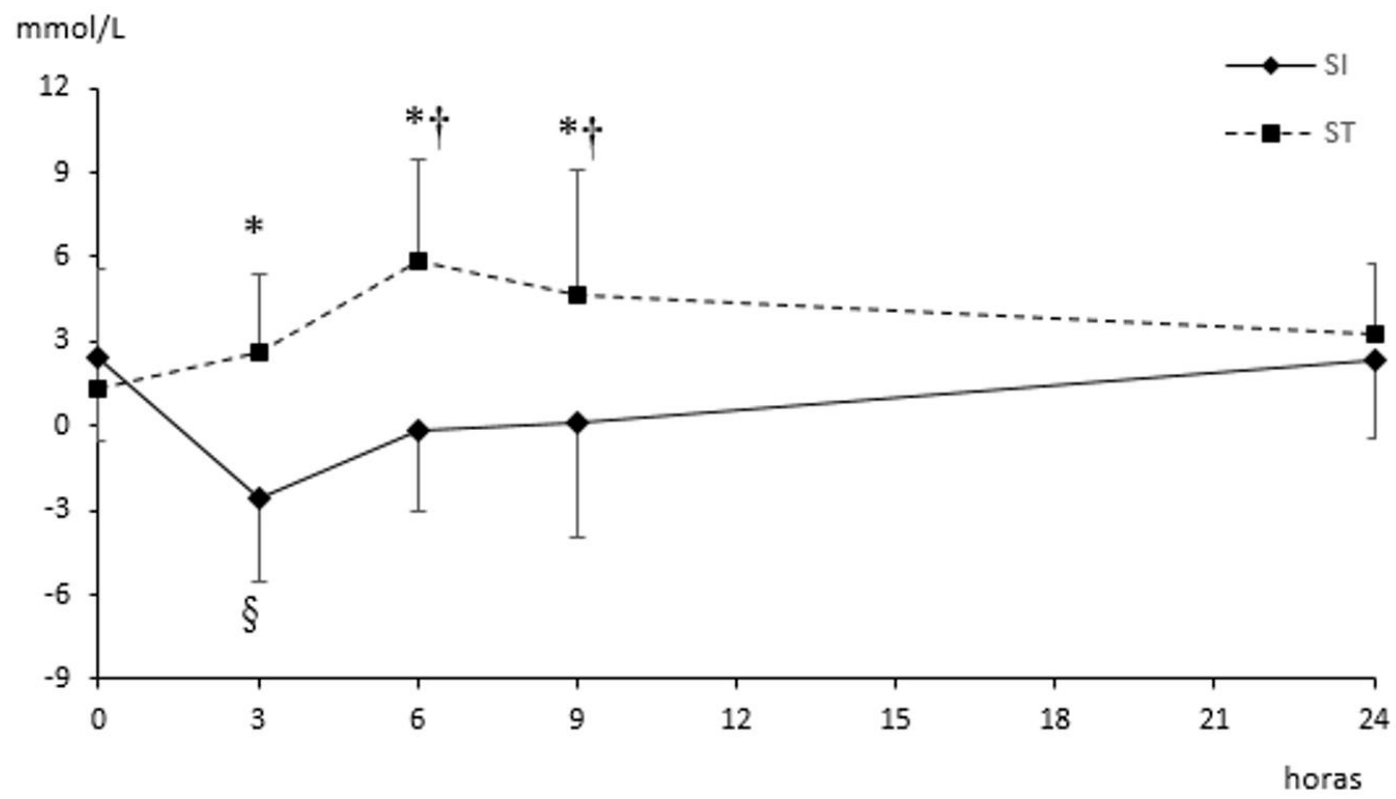

Figura 1. Variação do excesso de bases $(\mathrm{BE})$ em bovinos sadios que receberam infusão intravenosa de solução salina isotônica ( $\mathrm{SI} ; \mathrm{NaCl} 0,9 \%$ ) ou da solução eletrolítica testada (ST), em volume correspondente a $5 \%$ do peso corporal, com início na hora zero e término na hora três. (*) representa diferença entre as soluções ( $\mathrm{p}<0,05)$; $(\dagger)$ representa diferença em relação ao momento de início da infusão da ST ( $<<0,01$ ); ( $($ ) representa diferença em relação ao momento de início da infusão da SI $(\mathrm{p}<0,01)$. 
Tabela 1. Variações (média \pm desvio padrão) das variáveis hemogasométricas, dos eletrólitos e de variáveis bioquímicas em bovinos sadios que receberam infusão intravenosa de solução salina isotônica ( $\mathrm{SI} ; \mathrm{NaCl}$ $0,9 \%$ ) ou da solução eletrolítica testada (ST), em volume correspondente a $5 \%$ do peso corporal, com início na hora zero e término na hora três

\begin{tabular}{|c|c|c|c|c|c|}
\hline Solução & 0 hora & 3 horas & 6 horas & 9 horas & 24 horas \\
\hline \multicolumn{6}{|c|}{ pH } \\
\hline SI & $7,42^{A A} \pm 0,02$ & $7,37^{180} \pm 0,04$ & $7,41^{\mathrm{Bs}} \pm 0,02$ & $7,42^{\mathrm{Bs}} \pm 0,01$ & $7,39^{8=b} \pm 0,05$ \\
\hline ST & $7,41^{\wedge \mathrm{b}} \pm 0,03$ & $7,40^{\wedge \mathrm{b}} \pm 0,04$ & $7,44^{\wedge=b} \pm 0,03$ & $7,45^{\wedge \mathrm{s}} \pm 0,02$ & $7,43^{\mathrm{Aab}} \pm 0,02$ \\
\hline \multicolumn{6}{|c|}{$\mathrm{pCO}_{2}(\mathrm{mmHg})$} \\
\hline SI & $41,45^{\wedge a b} \pm 3,46$ & $40,51^{\wedge \mathrm{b}} \pm 4,12$ & $38,76^{\mathrm{Bb}} \pm 2,81$ & $38,38^{\wedge b} \pm 5,68$ & $45,81^{\wedge a} \pm 8,08$ \\
\hline ST & $41,30^{A x} \pm 3,89$ & $44,40^{\lambda x} \pm 6,18$ & $43,27^{\wedge s} \pm 2,92$ & $41,31^{\wedge s} \pm 4,13$ & $42,35^{\wedge a} \pm 3,73$ \\
\hline \multicolumn{6}{|c|}{$\mathrm{HCO}_{3}{ }^{-}\left(\mathrm{mmol}^{\prime} \mathrm{L}\right)$} \\
\hline SI & $26,77^{\wedge s} \pm 2,70$ & $22,77^{\mathrm{B} b} \pm 2,56$ & $24,36^{\mathrm{Bas}} \pm 2,51$ & $24,95^{\mathrm{Bab}} \pm 4,09$ & $27,23^{A x} \pm 2,83$ \\
\hline ST & $25,88^{\wedge \mathrm{b}} \pm 3,83$ & $27,87^{\wedge \Leftrightarrow} \pm 2,60$ & $30,15^{\wedge a} \pm 3,08$ & $28,56^{\text {A2b }} \pm 3,99$ & $27,57^{\wedge a b} \pm 2,36$ \\
\hline \multicolumn{6}{|c|}{$\mathrm{BE}(\mathrm{mmol} / \mathrm{L})$} \\
\hline SI & $2,42^{\wedge s} \pm 2,92$ & $-2,55^{\mathrm{Bb}} \pm 2,96$ & $-0,18^{\mathrm{Bes}} \pm 2,81$ & $0,15^{\mathrm{Bab}} \pm 4,09$ & $2,36^{\wedge x} \pm 2,81$ \\
\hline ST & $1,30^{\wedge \triangleright} \pm 4,32$ & $2,60^{\wedge 2 b} \pm 2,82$ & $5,89^{A A} \pm 3,53$ & $4,67^{\wedge \mathrm{a}} \pm 4,41$ & $3,30^{\wedge a b} \pm 2,44$ \\
\hline \multicolumn{6}{|c|}{$\mathrm{AG}(\mathrm{mmol} / \mathrm{L})$} \\
\hline SI & $14,78 \pm 3,13$ & $15,35 \pm 3,27$ & $16,61 \pm 1,52$ & $14,74 \pm 4,07$ & $16,17 \pm 1,93$ \\
\hline ST & $15,12 \pm 1,55$ & $14,01 \pm 1,06$ & $13,87 \pm 1,44$ & $14,65 \pm 2,70$ & $15,90 \pm 1,90$ \\
\hline \multicolumn{6}{|c|}{$\operatorname{SID}(\mathrm{mmol} / \mathrm{L})$} \\
\hline SI & $41,55^{\text {A2s }} \pm 3,99$ & $38,11^{\mathrm{Bb}} \pm 3,66$ & $40,97^{\mathrm{Bas}} \pm 3,14$ & $39,69^{\mathrm{Bb}} \pm 4,11$ & $43,41^{\wedge x} \pm 4,12$ \\
\hline ST & $41,00^{A s} \pm 3,34$ & $41,30^{\wedge \mathrm{a}} \pm 2,99$ & $43,55^{\wedge a} \pm 2,69$ & $43,21^{A a} \pm 3,91$ & $43,47^{\wedge s} \pm 2,57$ \\
\hline \multicolumn{6}{|c|}{$\mathrm{Na}^{+}(\mathrm{mmol} / \mathrm{L})$} \\
\hline SI & $137,47^{\wedge \mathrm{b}} \pm 3,63$ & $142,71^{\wedge a b} \pm 4,75$ & $143,81^{\wedge x} \pm 4,05$ & $144,06^{\wedge x} \pm 3,61$ & $140,56^{\text {Aab }} \pm 1,93$ \\
\hline ST & $137,82^{\wedge s} \pm 3,57$ & $139,56^{\wedge s} \pm 1,97$ & $144,44^{\wedge s} \pm 2,77$ & $143,62^{\wedge a} \pm 2,02$ & $141,03^{\wedge a} \pm 2,12$ \\
\hline \multicolumn{6}{|c|}{$\mathrm{K}^{+}(\mathrm{mmol} / \mathrm{L})$} \\
\hline SI & $3,64^{\text {Aab }} \pm 0,17$ & $3,41^{\wedge a b} \pm 0,35$ & $3,28^{\wedge \mathrm{b}} \pm 0,14$ & $3,38^{\wedge \mathrm{b}} \pm 0,23$ & $3,81^{\wedge s} \pm 0,22$ \\
\hline ST & $3,85^{\wedge s} \pm 0,33$ & $2,66^{\mathrm{Bb}} \pm 0,35$ & $2,54^{\mathrm{Bb}} \pm 0,33$ & $3,04^{\wedge \mathrm{b}} \pm 0,64$ & $3,70^{\wedge x} \pm 0,21$ \\
\hline \multicolumn{6}{|c|}{$\mathrm{Cr}(\mathrm{mmol} / \mathrm{L})$} \\
\hline SI & $99,11^{\wedge \mathrm{b}} \pm 1,91$ & $108,01^{\wedge a} \pm 3,36$ & $106,12^{\wedge x} \pm 2,43$ & $107,75^{\wedge s} \pm 2,45$ & $100,96^{\wedge b} \pm 2,87$ \\
\hline ST & $100,18^{\wedge s} \pm 3,48$ & $100,92^{\mathrm{Ba}} \pm 3,83$ & $102,95^{\mathrm{Ba}} \pm 3,31$ & $103,45^{\mathrm{Ba}} \pm 5,29$ & $100,80^{\wedge a} \pm 3,09$ \\
\hline \multicolumn{6}{|c|}{ Lactato $\mathbf{L}(\mathrm{mmol} / \mathrm{L})$} \\
\hline SI & $0,65^{\wedge s} \pm 0,68$ & $0,37^{\mathrm{B}_{3}} \pm 0,47$ & $0,20^{\wedge a} \pm 0,12$ & $0,30^{\wedge a} \pm 0,27$ & $0,66^{\wedge a} \pm 0,65$ \\
\hline $\mathrm{ST}$ & $1,01^{\wedge \mathrm{b}} \pm 1,95$ & $2,68^{\wedge s} \pm 1,46$ & $1,05^{\wedge b} \pm 0,91$ & $0,96^{\wedge b} \pm 0,61$ & $0,57^{A b} \pm 0,44$ \\
\hline \multicolumn{6}{|c|}{ Glicose $(\mathrm{mg} / \mathrm{dL})$} \\
\hline SI & $66,25^{\wedge_{s}} \pm 11,02$ & $63,87^{\mathrm{Ba}} \pm 10,34$ & $65,75^{\wedge a} \pm 6,31$ & $67,37^{\wedge a} \pm 11,33$ & $64,87^{\lambda a} \pm 10,26$ \\
\hline ST & $65,87^{\wedge b} \pm 9,71$ & $318,62^{\wedge x} \pm 130,74$ & $104,72^{\wedge b} \pm 69,62$ & $60,75^{\wedge \mathrm{b}} \pm 7,74$ & $62,50^{\wedge \mathrm{b}} \pm 10,90$ \\
\hline \multicolumn{6}{|c|}{ Cálcio (mg/dL) } \\
\hline SI & $9,62 \pm 0,34$ & $8,90 \pm 0,41$ & $9,19 \pm 0,30$ & $9,22 \pm 0,37$ & $9,44 \pm 0,46$ \\
\hline ST & $9,42 \pm 0,47$ & $9,06 \pm 0,41$ & $9,12 \pm 0,33$ & $9,17 \pm 0,60$ & $9,49 \pm 0,50$ \\
\hline \multicolumn{6}{|c|}{ Cálcio ionizado (mg/dL) } \\
\hline SI & $4,34 \pm 0,36$ & $4,31 \pm 0,54$ & $4,07 \pm 0,25$ & $4,32 \pm 0,36$ & $4,45 \pm 0,10$ \\
\hline ST & $4,33 \pm 0,34$ & $4,08 \pm 0,32$ & $3,80 \pm 0,51$ & $4,03 \pm 0,63$ & $3,96 \pm 0,44$ \\
\hline \multicolumn{6}{|c|}{ Magnésio (mg/dL) } \\
\hline SI & $2,21 \pm 0,40$ & $1,93 \pm 0,27$ & $2,00 \pm 0,27$ & $2,03 \pm 0,31$ & $2,08 \pm 0,33$ \\
\hline ST & $2,04 \pm 0,20$ & $2,03 \pm 0,34$ & $2,02 \pm 0,11$ & $2,02 \pm 0,15$ & $2,07 \pm 0,17$ \\
\hline \multicolumn{6}{|c|}{ Fósforo $(\mathrm{mg} / \mathrm{dL})$} \\
\hline SI & $6,47^{\wedge s} \pm 1,52$ & $5,32^{\wedge \mathrm{b}} \pm 1,73$ & $5,72^{\mathrm{Ab}} \pm 1,54$ & $5,63^{\mathrm{Aab}} \pm 1,80$ & $6,37^{\wedge a} \pm 1,62$ \\
\hline ST & $5,61^{\text {Aab }} \pm 0,86$ & $4,27^{\wedge \mathrm{b}} \pm 1,37$ & $4,43^{\wedge b} \pm 1,04$ & $5,70^{\wedge a b} \pm 0,90$ & $6,01^{\wedge a} \pm 0,55$ \\
\hline
\end{tabular}

${ }^{A, B}$ Representam diferenças entre as soluçðes no mesmo momento $(\mathrm{p}<0,05)$.

${ }^{a, b}$ Representam diferenças entre os momentos na mesma soluçăo $(p<0,05)$. 
A metabolização do lactato é rápida e a alcalinização é imediata em bovinos ${ }^{(7)}$. Nos animais estudados, a concentração de lactato no plasma estava aumentada $(\mathrm{p}<0,01)$ ao final da infusão da ST e retornou aos valores basais três horas após (Tabela 1). Os valores mais altos de $\mathrm{HCO}_{3}{ }^{-}$e de $\mathrm{BE}$ não foram observados ao término da infusão, mas sim três horas depois.

A alcalinização gerada pela ST pode ser considerada leve, a julgar pelo incremento do valor do BE. Administrada em volume correspondente a $5 \%$ do peso corporal, a ST elevou somente 4,6 mmol/L no valor médio do BE. Esse aumento pode ser considerado insuficiente para a correção dos estados de acidose metabólica de graus moderado a acentuado.

Quando uma solução eletrolítica é infundida na corrente sanguínea, a sua SID efetiva provoca alteração na SID plasmática, como reflexo das mudanças nas concentrações de $\mathrm{Na}^{+}$e $\mathrm{Cl}^{-}$do plasma ${ }^{(5)}$. As duas soluções infundidas no presente estudo diferem com relação à concentração destes íons. A SI possui concentrações equivalentes de $\mathrm{Na}^{+}$e de $\mathrm{Cl}^{-}$(154 mEq/L de cada íon), o que gera a SID efetiva igual a $0 \mathrm{mEq} / \mathrm{L}$. A ST possui $145 \mathrm{mEq} / \mathrm{L} \mathrm{de} \mathrm{Na}^{+}, 5 \mathrm{mEq} / \mathrm{L}$ de $\mathrm{K}^{+}$e $96 \mathrm{mEq} / \mathrm{L} \mathrm{de} \mathrm{Cl}^{-}$, o que aumenta a sua SID efetiva para $54 \mathrm{mEq} / \mathrm{L}$. Considerando que a SID plasmática dos indivíduos sadios é próxima de $40 \mathrm{mmol} / \mathrm{L}^{(5)}$, soluções que possuem SID efetiva alta têm capacidade alcalinizante ${ }^{(11)}$, enquanto as com SID efetiva baixa são acidificantes. Para cada $1 \mathrm{mEq} / \mathrm{L}$ de diminuição na SID plasmática ocorre um decréscimo de 0,013 no pH sanguíneo ${ }^{(12)}$. Essa interpretação também poderia explicar os efeitos acidificante da SI e alcalinizante da ST.

A SI aumentou as concentrações de $\mathrm{Na}^{+}(\mathrm{p}<0,01)$ e de $\mathrm{Cl}^{-}(\mathrm{p}<0,001)$ nos bovinos estudados (Tabela 1). E, ainda que os valores tenham se mantido dentro dos limites fisiológicos ${ }^{(13)}$, essa solução provocou hipercloremia relativa com consequente acidificação. A variação da SID plasmática, com redução discreta ao término da infusão $(\mathrm{p}<0,01)$, foi representativa desse evento. A ST, por outro lado, não alterou as concentrações plasmáticas de $\mathrm{Na}^{+}$ou de $\mathrm{Cl}^{-}$nem tampouco a SID (Tabela 1). Isso se deve ao fato de a sua composição eletrolítica ser relativamente parecida com a do plasma dos bovinos $^{(13)}$, o que diminui a probabilidade de induzir desequilíbrios eletrolíticos iatrogênicos. Comparada à SRL, a ST não poderia ser considerada essencialmente uma solução balanceada. No entanto, em comparação à SI, a sua composição é muito mais próxima da situação fisiológica.

Apesar disso, os bovinos apresentaram hipopotassemia transitória $(\mathrm{p}<0,001)$ quando receberam a ST, evidente ao término da infusão e três horas após (Tabela 1). A hipopotassemia pode ocorrer em virtude do movimento do $\mathrm{K}^{+}$do espaço líquido extracelular para o intracelular, o que não reflete a depleção verdadeira do $\mathrm{K}^{+}$corpóreo $^{(14)}$. A hiperinsulinemia decorrente da hiperglicemia gera movimento insulino-dependente do $\mathrm{K}^{+}$para dentro da célula que, somada à expansão do volume plasmático e à alcalinização, pode contribuir para diminuir a concentração de $\mathrm{K}^{+}$no sangue ${ }^{(15)}$. Como o efeito alcalinizante da ST foi baixo e a elevação iatrogênica da glicemia foi acentuada ao final da infusão, as alterações nas concentrações de $\mathrm{K}^{+}$observadas podem ser atribuídas principalmente à hiperglicemia. Este efeito foi transitório e em 24 horas as concentrações retornaram aos valores iniciais.

A presença de 50g/L de dextrose na ST provocou hiperglicemia transitória ( $\mathrm{p}<0,001)$, sendo os valores máximos ao final da infusão e ainda elevados três horas após (Tabela 1). Deve-se salientar que os bovinos estudados eram saudáveis. É provável que, quando administrada em animais hipoglicêmicos, esta solução não cause hiperglicemia tão acentuada e, consequentemente, interfira 
menos com o equilíbrio do $\mathrm{K}^{+}$.

Ainda que não exista consenso sobre o valor mínimo crítico para a concentração de $\mathrm{K}^{+}$no plasma de bovinos, admite-se, de forma geral, que varie de 2,2 a $2,5 \mathrm{mmol} / \mathrm{L}^{(14)}$. Os bovinos estudados aproximaram-se desse valor crítico no final da infusão e três horas depois e não manifestaram qualquer sinal, mesmo leve, compatível com a síndrome hipopotassêmica descrita para essa espécie $^{(14)}$. Deve-se destacar que, curiosamente, os bovinos usados no estudo, apesar de sadios, possuíam concentrações plasmáticas médias iniciais de $\mathrm{K}^{+}$ligeiramente abaixo do limite inferior fisiológico de $3,9 \mathrm{mmol} / \mathrm{L}^{(13)}$. Isso pode ter contribuído para a magnitude da hipopotassemia observada após a infusão. Teoricamente, a infusão de volumes maiores da ST em bovinos hipopotassêmicos poderia ser considerada potencialmente desvantajosa. Entretanto, isso depende, supostamente, do efeito hiperglicemiante, mais provável em bovinos que possuam normo ou hiperglicemia antes de receberem a infusão.

As duas soluções estudadas não provocaram alterações na calcemia (tanto $\mathrm{Ca}$, quanto $\mathrm{Ca}^{++}$) e na magnesemia, mas geraram redução discreta e transitória da fosfatemia, provavelmente devido à hemodiluição iatrogênica. $\mathrm{O}$ AG também não se alterou mesmo em face da expansão do volume plasmático no caso das duas soluções e da hiperlactatemia no caso da ST.

Não foram observadas alterações no exame físico dos bovinos estudados. Independente da solução infundida, os parâmetros clínicos não variaram durante e após a infusão intravenosa $(\mathrm{p}>0,05)$ e mantiveram-se dentro dos limites fisiológicos admitidos para a espécie ${ }^{(10)}$. Isso indica que a ST é, muito provavelmente, uma solução eletrolítica segura para uso terapêutico em bovinos. Os desequilíbrios iatrogênicos observados com a infusão da ST foram transitórios, desaparecendo seis horas após o término da infusão, e não foram acompanhados por qualquer tipo de perturbação aparente da saúde.

\section{Conclusões}

Com base nos resultados, pode-se concluir que a solução testada (ST) possui potencial alcalinizante reduzido e sua utilização em bovinos é segura. A hiperglicemia e a hipopotassemia iatrogênicas podem ser desvantajosas quando a solução for administrada em animais não hipoglicêmicos e hipopotassêmicos, mas são efeitos transitórios.

\section{Agradecimentos}

Aos acadêmicos de Medicina Veterinária da UEL Anamaria Vedovato, João Fernando Zamariola Sanches, Lais Fernanda Sargi, Mayara Cardoso dos Anjos, Nathali Adrielli Agassi de Sales, Rafael Cascales e Stefany Lia Oliveira Camilo pelo auxílio na execução das atividades da fase experimental. 


\section{Referências}

1. Constable P. Fluid and electrolyte therapy in ruminants. Vet Clin North Am Food Anim Pract. 2003 Nov; 19(3):557-97.

2. Ribeiro Filho JD, Gimenez AM, Fonseca EF, Dantas, WMF, Oliveira TT. Hidratação enteral em bovinos: avaliação de soluções eletrolíticas isotônicas administradas por sonda nasogástrica em fluxo contínuo. Ciência Rural. 2011 fev, 41(2):285-290.

3. Berchtold J. Treatment of calf diarrhea: intravenous fluid therapy. Vet Clin North Am Food Anim Pract. 2009 Mar; 25(1):73-99.

4. Roussel AJ. Fluid therapy in mature cattle. Vet Clin North Am Food Anim Pract. 2014 Jul; 30(2): 429439.

5. Constable PD. Acid-base assessment: when and how to apply the Henderson-Hasselbalch equation and strong ion difference theory. Vet Clin North Am Food Anim Pract. 2014 Jul; 30(2): 295-316.

6. Leal MLR, Maruta CA, Ortolani EL. Use of bicarbonate and lactate L for correction of systemic metabolic acidosis in cattle with acute rumen lactic acidosis. Arq Bras Med Vet Zootec. 2007 Aug; 59(4):971-76.

7. Cosenza M, Pereira PFV, Fernandes LI, Dearo ACO, Flaiban KKMC, Lisbôa JAN. Efeito da solução de ringer com lactato sobre os equilíbrios hidroeletrolítico e acidobase de equinos, ovelhas e bezerros sadios. Ciência Rural. 2013 Dez; 43(12):2247-53.

8. Brasil. Ministério da Agricultura, Pecuária e Abastecimento, Secretaria de Defesa agropecuária, Instrução Normativa n²6, de 16 de setembro de 2005. [Acesso 19 fevereiro 2013]. Disponível em: http://sistemasweb.agricultura.gov.br/sislegis/action/detalhaAto.do?method=visualizarAtoPortalMapa\&chav $\mathrm{e}=1872912962$

9. Pereira PFV, Bessegatto JA, Bregadioli GC, Camilo SL, Sales NAA, Flaiban KKMC, Lisbôa JAN. Effects of a new intravenous electrolyte solution for veterinary therapy on the electrolyte and acid-base balances of healthy horses. Ciência Rural. 2016 Ago; 46(8):1479-85.

10. Dirksen G, Gründer HD, Stöber M. Rosenberger: Exame Clínico dos Bovinos. Rio de Janeiro: Guanabara Koogan, 3ed., 1993. 420p.

11. Muller KR, Gentile A, Klee W, Constable PD. Importance of effective strong ion difference of an intravenous solution in the treatment of diarrheic calves with naturally acquired acidemia and strong ion (metabolic) acidosis. J Vet Intern Med. 2012 May-Jun; 26(3):674-83.

12. Constable PD, Stampfli HR, Navetat H, Berchtold J, Schelcher F. Use of a quantitative strong ion approach to determine the mechanism for acid-base abnormalities in sick calves with or without diarrhea. $\mathbf{J}$ Vet Intern Med. 2005 Jul-Aug; 19(4):581-9.

13. Carlson GP, Bruss M. Fluid, eletrolyte, and acid-base balance. In: Kaneko JJ. Clinical biochemistry of domestic animals. 6th ed. Burlington: Elsevier; 2008. p. 529-559.

14. Sattler N, Fecteau G. Hypokalemia syndrome in cattle. Vet Clin North Am Food Anim Pract. 2014 Jul; 30(2): 351-7.

15. Grunberg W, Hartmann H, Burfeind O, Heuwieser W, Staufenbiel R. Plasma potassium-lowering effect of oral glucose, sodium bicarbonate, and the combination thereof in healthy neonatal dairy calves. J Dairy Sci. 2011 Nov; 94(11):5646-55. 